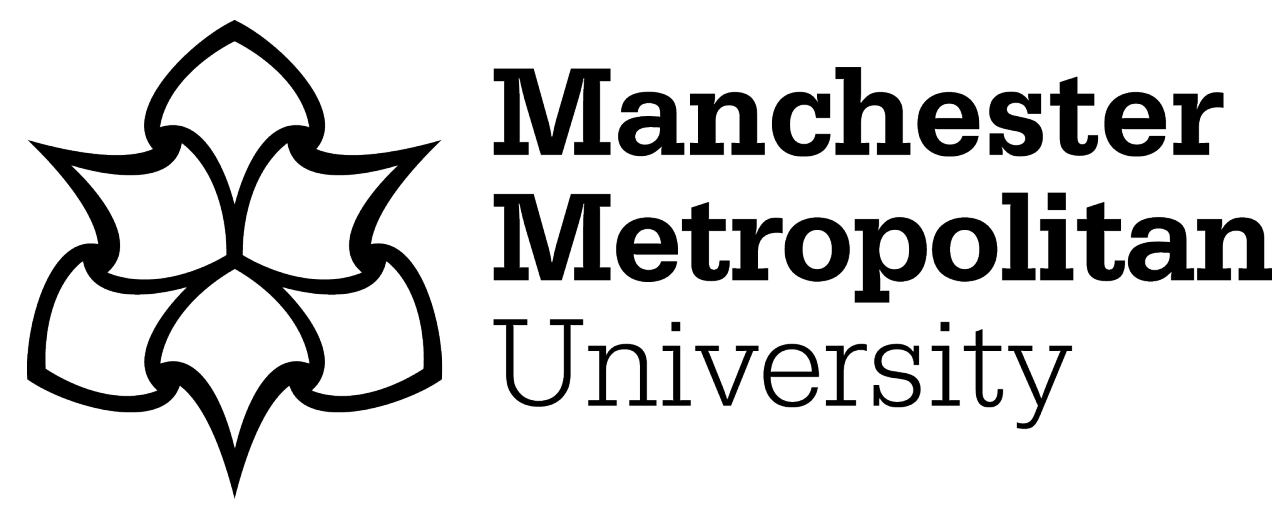

Charlotte, Blease, Annoni, Marco and Hutchinson, Anthony (2018) Editors' Introduction to Special Section on Meaning Response and the Placebo Effect. Perspectives in Biology and Medicine, 61 (3). pp. 349-352. ISSN 0031-5982

Downloaded from: https://e-space.mmu.ac.uk/621530/

Version: Accepted Version

Publisher: Johns Hopkins University Press

Please cite the published version 


\title{
EDITORS' INTRODUCTION TO SPECIAL SECTION ON MEANING Response AND THE Placebo EFFECT
}

\author{
Charlotte Blease, Marco AnNoni, and Phil hutchinson
}

\begin{abstract}
$\mathrm{O}_{\mathrm{i}}$ VER 200 YEARS AGO, DOCTORS' most effective tools were typically not found in their medical bags. Indeed, most treatments in the history of medicine have, until relatively recently, caused more harm than good. Prior to the biomedical revolution in the late 19th century, doctors' most reliable and effective instruments of healing were their skills of communication with patients and an aptitude for a positive and supportive bedside manner. Bearing out this portrait of medicine, Thomas Jefferson, writing in 1807, noted that "one of the most successful physicians I have ever known has assured me that he used more bread pills, drops of colored water, and powers of hickory ashes, than of all other medicines put together" (qtd. in De Craen et al. 1999, 511). Jefferson referred to these skills of beneficent persuasion as a "pious fraud." Exactly one hundred years later, in 1907, Mark Twain drew similar observations: "Physicians cure many patients with a bread pill; they know that where the disease is only a fancy, the patient's confidence in the doctor will make the bread pill effective."

Two major developments would downgrade the value of the interpersonal, doctor-patient relationship in professional medicine. The first development was the biomedical revolution. A wave of scientific medical success stories during the latter half of the 19th century would lead to a substantially more effective and reliable treatments and techniques than doctors had ever before employed. Encompassing a range of significant developments for public health and medical
\end{abstract}


care, the biomedical revolution included the emergence of the germ theory of disease, the use of anesthetics and antiseptics in surgery, and the development of vaccines. One consequence of these developments was the incorporation of determinedly scientific tools and training into biomedical education and clinical practice, with the result that the value of interpersonal, healing relationships between doctors and patients was devalued. In keeping with this idea, the Father of Modern Medicine, Canadian physician William Osler (1849-1919), acclaimed for putting medicine on a scientific footing and reforming medical education, taught that the temperament of the physician ought to be one of "detached concern." Emphasizing the medical virtue of "Aequanimitas," Osler placed a premium on scientific objectivity and a composed, steady, "clinical" demeanor in all patient interactions.

The second major development that indirectly devalued interpersonal doctor-patient interactions occurred in the mid- to late-20th century: the emergence of the randomized controlled trial (RCT). The goal of RCTs is to evaluate whether a drug or intervention is effective. Patients are randomly allocated to one of two groups: the "verum" or treatment that is under scrutiny, and a control group. The control group may receive no treatment or a placebo: the placebo is intended to serve as a yardstick; the goal is to eliminate all of the therapeutic "noise" that can arise when patients enroll in clinical trials, including any salubrious effects of healing rituals, medical paraphernalia, or the positive effects of interacting with health-care practitioners, as well as the natural progression of illness.

Even when the placebo pill or treatment is a sham, patients may experience health benefits from their participation in clinical trials. However, RCTs- the gold standard of clinical research - seek to determine whether the treatment under scrutiny is effective over and above any positive effects of participating in trials, and of receiving placebo interventions. They therefore aim to eliminate the effects of human interactions, thus indirectly (and perhaps ironically) further discrediting, or at least distracting from, the potential therapeutic value of doctor-patient interactions, including factors associated with the context of care.

However, somewhat paradoxically, RCTs have also helped identify the importance of placebo effects in such a way as to make them a respectable subject for study. The same experimental design of the typical RCT, which is used to test for the effectiveness of an innovative treatment, can be likewise deployed to the comparison of a placebo and a no-treatment group. One goal associated with this research is to harness the significant medical effects of the social practices of doctor-patient interactions to improve patient outcomes.

This Special Section aims to take the marginalized factors relating to practitioner-patient interactions out of the shadows of biomedicine and shine a spotlight on them. How should we conceive of any positive (or indeed, negative) effects that arise not from biomedical interventions, but from the rituals of healing and from human interactions? The phenomenon that we have vaguely described, 
and intentionally avoided pinning down, is nowadays commonly referred to as "placebo effect(s)" (Evers et al. 2018). But this terminology is still the subject of debate and controversy: some scholars argue that the term "placebo effect" should be conserved; others contend that it is deeply problematic and should be revised or reconceived; still others propose that it should be dropped from medical vocabulary altogether.

This Special Section focuses on one such proposal for reconceiving and redefining placebo effects. Howard Brody $(1980,2000)$ and Daniel Moerman (2002; Moerman and Jonas 2002) both independently argue that "meaning models" or the "meaning response" help to explain the salubrious effects observed in both the typical practitioner-patient interaction as well as in control groups in clinical trials. For example, Moerman proposes that the "meaning response" is "the psychological and physiological effects of meaning in the treatment of illness" (Moerman 2002, 14). On these distinctive frameworks, it is the meaning of treatments and health-care interactions for patients that can give rise to significant therapeutic effects.

While the respective publications by Brody and by Moerman remain the most highly cited in the field of placebo studies, the term meaning is not universally preferred, and it has fallen out of favor among placebo researchers-even while debate still persists about how best to define "placebo effects." Yet within other spheres of health-care research-for example, clinical psychology and narrative medicine-Moerman's term "meaning response" is still extensively used as a term of art.

To date, however, there has been no systematic, scholarly evaluation of the terms "meaning model" and "meaning response." This Special Section therefore aims to fulfill a neglected gap in health-care research by reflecting critically on the following themes: what might meaning mean in the realm of medicine and health care? Do such concepts add value to clinical research? Should we reconceive "placebo effects" via the concept of "meaning responses" or "meaning models"? And does the concept of meaning within patients' health-care experiences advance our understanding of therapeutic processes in other domains, such as clinical psychology? These- and other questions - are the central focus of this fresh reflection on Brody's and Moerman's work.

Most of the contributions in this collection emerged out of a multidisciplinary workshop hosted by Phil Hutchinson at Manchester Metropolitan University (MMU) in April 2016. The workshop (attended by Daniel Moerman) included papers by philosophers and psychologists, aimed at reflecting, specifically, on Moerman's concept of the "meaning response." A second event-the inaugural conference of the Society for Interdisciplinary Placebo Studies-held at the University of Leiden in April 2017, strengthened the cross-disciplinary conversations that arose at that MMU workshop, directly leading to additional papers included within this collection. 
The Special Section opens with contributions from the pioneers in "meaning" in medicine. The collection begins with a new paper on the original meaning model proposed by Howard Brody. This is followed by an article by Phil Hutchinson and Daniel Moerman, who weigh up three candidate explanations for "placebo responses": classical conditioning, response expectancy, and Moerman's own meaning response. Next is a "critical (and cautiously optimistic)" appraisal of Moerman's meaning response theory by philosophers Marco Annoni and Charlotte Blease. The Special Section then includes a range of distinctive papers aimed at describing and evaluating the variety of conceptualizations of placebo effects in the literature. The first of these papers reviews the variety of frameworks proposed for placebo effects, emphasizing why psychoneurobiological aspects of placebo phenomena provide a reliable tool in clinical practice. Next, a contribution by philosopher Andrew Turner proposes that any competing conceptualizations of placebo effects (including meaning models and the meaning response) need to be held accountable to their potential practical value within medical research and practice. Finally, taking a different perspective, Charlotte Blease argues that conceptual disagreement in the field of placebo studies is overstated: drawing on insights from Thomas Kuhn, she argues that-appearances to the contrary - there is considerable underlying consensus about placebo concepts among researchers.

We thank the contributors for their stimulating contributions, and the Editors of Perspectives in Biology and Medicine for their excellent editorial guidance. We are confident that this Special Section will stimulate further cross-disciplinary discussion and renewed debate on the meaning response, inspire deeper reflection on conceptual disputes in placebo studies, and encourage more discussion about the role of meaning within medicine and psychotherapy.

\section{REFERENCES}

Brody, H. 1980. Placebos and the Philosophy of Medicine Clinical, Conceptual, and Ethical Issues. Chicago: University of Chicago Press.

Brody, H. 2000. "The Placebo Response: Recent Research and Implications for Family Medicine." J Fam Pract 49: 649-54.

De Craen, A. J., et al. 1999. "Placebos and Placebo Effects in Medicine: Historical Overview." J R Soc Med 92 (10): 511-15.

Evers, A. W. M., et al. 2018. "Implications of Placebo and Nocebo Effects for Clinical Practice: Consensus of a Group of Experts." Psychother Psychosom. DOI: $10.1159 / 000490354$.

Moerman, D. 2002. Meaning, Medicine, and the "Placebo Effect." Cambridge: Cambridge University Press.

Moerman, D., and W. B. Jonas. 2002. "Deconstructing the Placebo Effect and Finding the Meaning Response." Ann Intern Med 136 (6): 471-76.

Twain, M. 1907. Christian Science. N.p.: Hewlett Press, 2014. 